\title{
Context-Aware Systems and Applications (ICCASA 2018) and Nature of Computation and Communication (ICTCC 2018)
}

\author{
Phan Cong Vinh ${ }^{1}$ \\ Published online: 3 October 2018 \\ (C) Springer Science+Business Media, LLC, part of Springer Nature 2018
}

\section{Foreword}

This special issue, with six papers invited and extended from selected contributions to The 7th International Conference on Context-Aware Systems and Applications (ICCASA 2018) and The 4th International Conference on Nature of Computation and Communication (ICTCC 2018) held in Viet Tri City of Vietnam, will serve as a reference material for researchers, scientists, professionals and students in computer science and computer engineering as well as developers and practitioners in computing and networking systems design by providing them with state-of-the-art research findings and future opportunities and trends. These papers include some various aspects of context-awareness and nature of computation and communication reflected in this special issue. In particular, the special issue covers various contributions of context-awareness and nature of computation and communication as follows:

Paper 1 by Abdur Rakib and Ijaz Uddin shows that over the last few years, context-aware computing has received a growing amount of attention among the researchers in the IoT and ubiquitous computing community. In principle, context-aware computing transforms physical environment into smart spaces by sensing surrounding environment and interpreting the situation of the user. This process involves three major steps: context acquisition, context modelling, and context-aware reasoning. Among other approaches, ontology-based context modelling and rule-based context reasoning are widely used techniques to enable semantic interoperability and interpreting user situations. However, implementing rich context-aware applications that perform reasoning on resource-bounded mobile devices is quite challenging. In this paper, authors present a context-aware systems development framework for smart

Phan Cong Vinh

pcvinh@ntt.edu.vn

1 Nguyen Tat Thanh University, 300A Nguyen Tat Thanh Street, Ward 13, District 4, Ho Chi Minh City, Vietnam

spaces, which includes a light weight efficient rule engine and a wide range of user preferences to reduce number of rules while inferring personalized contexts. This shows rules can be reduced in order to optimize the inference engine execution speed, and ultimately to reduce total execution time and execution cost.

Paper 2 by Shuangqing Xiang, Xi Wu, Huibiao Zhu, Wanling Xie, Lili Xiao and Phan Cong Vinh points out that Software-Defined Networking (SDN) is an emerging networking paradigm that provides flexible network programmability and reduces the complexity of network management and control. OpenFlow Protocol is the best-known southbound interface of SDN. As one of the most popular OpenFlow controllers, Floodlight is famous for its excellent basic modules, which are used by many other mainstream controllers. Due to the popularity and widespread use of the Floodlight's modules, it is important to analyze them in a formal famework. In this paper, authors focus on six modules of Floodlight that are mainly responsible for pushing decisions and discovering topology. In order to verify whether these modules meet two important requirements, including Decision Pushed Correctly and Rule Installed Once, authors propose a system model that formalizes these modules as well as hosts and switches using Communicating Sequential Processes (CSP). Moreover, we add two types of attacks: Link Fabrication and Host Hijacking, which aim at poisoning the topology information collected by a controller, to construct a second system model. Finally, authors implement these two system models in Process Analysis Toolkit (PAT), a model checker, to verify whether the models cater for four assertions based on two requirements and if Floodlight is vulnerable to these two attacks.

Paper 3 by Phuong L. Vo, Tuan-Anh Le, Nguyen H. Tran presents that today, most of the smart devices are equipped with more than one network interfaces. A multipath congestion control protocol that exploits different paths to transmit data will improve the throughput and high-availability. Many multipath congestion control protocols have been developed from the corresponding single-path congestion control protocols. Most of them are loss-based algorithms, hence, they do 
not well utilize the bandwidth in high bandwidth-delay product (BDP) connections due to the conservative congestion control (additive increase - multiplicative decrease). From the single-path Fast TCP, authors develop a multipath congestion control protocol, called mFast, for high BDP connection. mFast uses queueing delay to measure the congestion as Fast TCP does. The framework is based on a network utility maximization model for multipath ows. The features of mFast such as load-balancing, TCP friendliness, and throughput improvement are verified via analysis and extensive simulations.

Paper 4 by Hong Anh Le, Anh Tu Bui, Ninh-Thuan Truong shows that recently, the number of mobile users grow enormously. Even though hardware technologies have been taken to many advantages, which aim at increasing the battery capacity, people are still facing with the problem of battery shortage. An application that runs functionally correct and has a friendly graphic user interfaces still causes users frustrated if it drains the battery. In parallel with increasing the energy storage capability of mobile device, optimizing the source code to reduce power consumption is an emerging topic. Authors present a new approach to modeling and evaluating power consumption of mobile applications. First, authors introduce new definitions of power states and a power consumption automaton (PCA) for a hardware component. In the next step, authors propose algorithms to optimize and merge these into an unified automaton. In order to estimate the power consumption amount, the power automaton is refined with time aspect and power coefficients. Finally, authors develop a support tool, which is a plug-in for Android studio and IntelliJ for visualizing the power machines and estimating the accumulated power consumption of an application use-case.

Paper 5 by Wanling Xie, Huibiao Zhu, Xi Wu, Phan Cong Vinh points out that node mobility, as one of the most important features of Wireless Sensor Networks (WSNs), may affect the reliability of communication links in the networks, leading to abnormalities and decreasing the quality of service provided by WSNs. The mCWQ calculus (i.e., CWQ calculus with mobility) is recently proposed to capture the feature of node mobility and increase the communication quality of WSNs. In this paper, authors present a proof system for the $\mathrm{mCWQ}$ calculus to prove its correctness. Our specifications and verifications are based on Hoare Logic. In order to describe the timing of observable actions, authors extend the assertion language with primitives. And terminating and nonterminating computations both can be described in the proof system. Authors also give some examples to illustrate the application of the proof system.

Paper 6 by Attasit Patanasiri, Donyaprueth Krairit aims to study consumers' purchase intention on web-based and social-based B2C ecommerce platforms. This research is one of the first empirical studies to employ the Stimulus-
Organism-Response Model (S-O-R Model) as a main research model. Authors found six findings which contrast with the previous literature. First, the two-way communication tools provided by the platform are not powerful enough to significantly affect the emotional needs of online consumers. Second, the stimulus that affect user's interpretation of online information is the two-way communication tools provided by the platform. Third, the risk perceived by a consumer in contemplating a particular purchase action does not significantly affect the emotional needs of consumers. Fourth, the stimulus that affects users' curiosity aroused while interacting with the platform is the ability of the platform to enhance user participation in the platform's activities. Fifth, consumers' emotional needs significantly affect online consumers' purchase intention. Finally, the degree of users' attention while using the platform significantly affects consumers' purchase intention.

We owe our deepest gratitude to Prof. Nguyen Manh Hung - Chairman and Rector of Nguyen Tat Thanh University in Vietnam for his useful support, especially to all the authors for their valuable contribution to this special issue and their great efforts, and also to the referees for ensuring the high quality of the material presented here. All of them are extremely professional and cooperative. We wish to express our thanks to the Editor-in-Chief, Prof. Imrich Chlamtac, for his important assistance with the process of assembling the special issue.

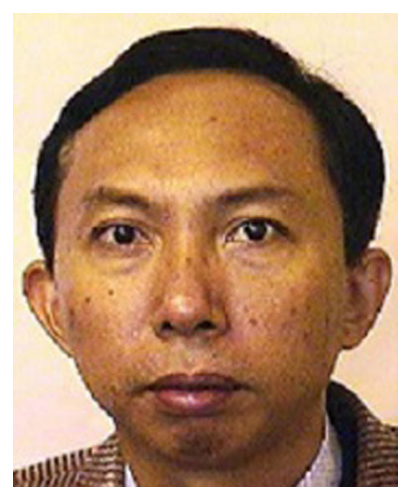

Phan Cong Vinh received a PhD in computer science from London South Bank University (LSBU) in the United Kingdom. He finished his $\mathrm{PhD}$ dissertation with the title of "Formal Aspects of Dynamic Reconfigurability in Reconfigurable Computing Systems" at LSBU where he was affiliated with the Center for Applied Formal Methods (CAFM) at the Institute for Computing Research (ICR). At present, he is an Associate Professor of Nguyen Tat Thanh University (NTTU) to take on the responsibility of a senior research scientist. He has been author or co-author of many refereed contributions published in prestigious journals, conference proceedings or edited books. He is editor of three books titled, "Autonomic Networking-onChip: Bio-Inspired Specification, Development and Verification" (CRC Press, 2012), "Formal and Practical Aspects of Autonomic Computing and Networking: Specification, Development and Verification" (IGI Global, 2011) and "Nature-Inspired Networking: Theory and Applications" (CRC Press, 2018). He has served on many conference program committees and has been general or technical (co)chair and (co)organizer of several international conferences such as ICCASA and ICTCC series. His research interests center on all aspects of formal methods in computing, context-awareness, nature of computation and communication, and applied categorical structures in computer science. 